\title{
Rapid Eye Movement Sleep Behaviour Disorder and its Association with Parkinson's Disease
}

\author{
An Expert Interview with Ambra Stefani \\ Department of Neurology, Medical University of Innsbruck, Innsbruck, Austria
}

DOl: https://doi.org/10.17925/ENR.2019.14.1.16

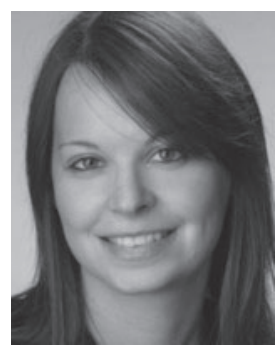

\begin{abstract}
Ambra Stefani
Ambra Stefani was born in Italy, where she performed her medical training at the University of Verona. After that she moved to Innsbruck, Austria, where she is currently working as resident and PhD student in the Department of Neurology, Medical University of Innsbruck, Austria. Ambra Stefani has been a member of the sleep research group, led by Prof. Birgit Högl, since 2013. Her research interest is mainly focused on movement disorders in sleep, in particular, (a) restless legs syndrome, with a special interest in better understanding the pathophysiological mechanisms of this disease; and (b) rapid eye movement (REM) sleep behaviour disorder, with a broad research spectrum ranging from improvement of diagnosis and screening of this condition, to the link between REM sleep behaviour disorder and neurodegeneration, including also the prodromal phases of REM sleep behaviour disorder.
\end{abstract}

\section{Keywords}

Rapid eye movement sleep behaviour disorder, parasomnia, Parkinson's disease

Disclosure: Ambra Stefani has no conflicts of interest to declare in relation to this article.

Review Process: This is an expert interview and, as such, has not undergone the journal's standard review process.

Acknowledgements: Medical writing assistance was provided by Katrina Mountfort Freelance Writer for Touch Medical Media, and was supported by Touch Medical Media.

Authorship: The named author meets the criteria of the International Committee of Medical Journal Editors for authorship for this manuscript, takes responsibility for the integrity of the work as a whole and has given final approval for the version to be published.

Received: 26 March 2019

Published Online: 19 July 2019

Citation: European Neurological Review. 2019;14(1):16-7

Corresponding Author: Ambra Stefani,

Department of Neurology, Anichstrasse 35, 6020

Innsbruck, Austria. E: ambra.stefani@i-med.ac.at

Support: No funding was received in

the publication of this article.
$\mathrm{R}$ apid eye movement (REM) sleep behaviour disorder (RBD) is a parasomnia characterised by movements and vocalisations during REM sleep that is caused by a loss of REM sleep atonia. ${ }^{1,2}$ Several studies in the last decades showed that RBD is, in most cases, an early manifestation of an alpha-synuclein related neurodegenerative disorder such as Parkinson's disease $(\mathrm{PD}){ }^{3}$

In an expert interview, Ambra Stefani discusses the diagnosis of RBD and its associations with neurological disease.

\section{Q. What is RBD and how prevalent is it?}

RBD is a REM sleep parasomnia and is characterised by movement and vocalisation during REM sleep, which are related to dream content. In order to diagnose RBD, polysomnography (PSG) is needed to demonstrate the presence of REM sleep without atonia, which is an excessive electromyographic activity during REM sleep., ${ }^{3,4}$ Interestingly, it is uncertain whether dreams cause the movements in RBD or if the movements due to loss of REM atonia produce a sensory stimulus that is responsible of the dream content. The question about prevalence is important because current data estimate a low population prevalence, between $0.3-1.2 \%{ }^{2,5}$ However, we are still lacking good epidemiological studies using video-PSG, so the real prevalence might be underestimated.

\section{Q. How is RBD associated with neurological disease, for example Parkinson's disease and associated neurological disorders?} Isolated REM sleep behaviour disorder is now recognised as a prodromal phase of alpha-synucleinopathy. Several studies showed that over time (even more than 10 or 15 years after diagnosis of RBD) more than $80 \%$ of these patients develop an overt alpha-synucleinopathy - which can be PD, dementia with Lewy bodies or multiple system atrophy. ${ }^{6}$ It is estimated that 10 years after the diagnosis of isolated RBD, approximately $45 \%$ of patients with RBD develop dementia with Lewy bodies, 45\% develop PD, and 5\% multiple system atrophy, whereas around $5 \%$ do not convert. However, even these patients with long-standing isolated RBD present several biomarkers of alpha-synuclein related neurodegeneration. ${ }^{7}$ This high conversion rate leads, not only to the inclusion of RBD as a risk marker in the Movement Disorders Society (MDS) research criteria for prodromal PD, but also to the assignment of the highest positive likelihood ratio (130) to PSG-proven RBD among all risk markers for PD. ${ }^{8}$

\section{Q. What factors should be considered in the differential diagnosis of RBD?}

It is important to realise that the diagnosis of RBD is not simple and is not possible based only on clinical interview of the patient, even for sleep experts. This is why even validated questionnaires for RBD 
demonstrated low sensitivity and specificity outside the context of validation studies, since RBD mimics may lead to false positives and unawareness of the disease to false negatives. In cases of suspected RBD, it is essential to perform video-PSG to make a definite diagnosis and to exclude other conditions which can mimic RBD, such as periodic leg movement during sleep, which can be violent, thus resembling RBD, or movement at the end of apnoea in patients with obstructive sleep apnoea syndrome.

\section{Q. What can be done to improve the recognition and diagnosis of this disorder?}

There is a need for increased awareness among the general population because patients who move during the night while experiencing nightmares might not mention this to their doctor. Awareness should also be increased among general practitioners as patients with suspected RBD need to be referred to a sleep laboratory to perform video-PSG, including electromyography of the upper extremities, to confirm the diagnosis of RBD and exclude mimics.

\section{Q. What current and emerging treatments are available for this disorder?}

The recommended treatments for RBD are melatonin and clonazepam. These are symptomatic treatments to lower the number and intensity of movements during the night, in order to reduce the risk of injury. ${ }^{9}$ As RBD is now recognised as an early phase alpha-synucleinopathy, studies testing neuroprotective treatment, which should prevent conversion to conditions such as PD, are expected.

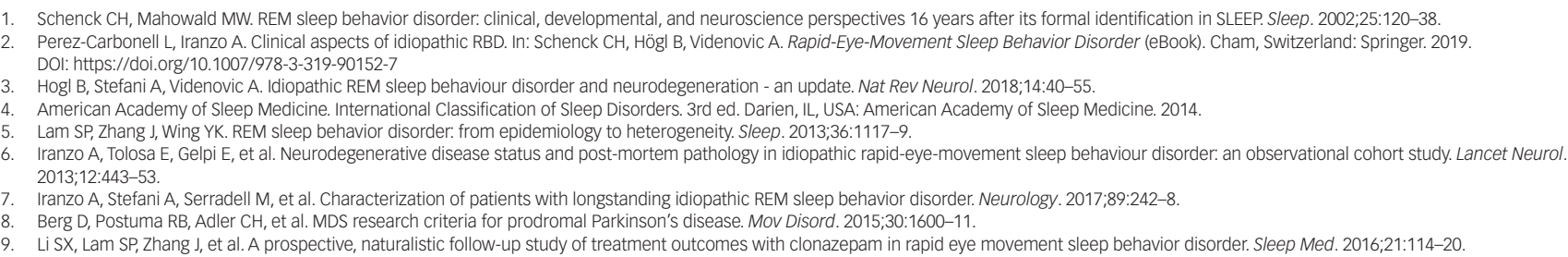

9. Li SX, Lam SP, Zhang J, et al. A prospective, naturalistic follow-up study of treatment outcomes with clonazepam in rapid eye movement sleep behavior disorder. Sleep Med. 2016;21:114-20. 